\title{
El aprendizaje servicio en universidades de Buenos Aires: avanzando hacia su institucionalización
}

\section{Monike Gezuraga}

Universidad del País Vasco, España

\section{María Alejandra Herrero}

Universidad de Buenos Aires, Argentina. Centro Latinoamericano de Aprendizaje y Servicio Solidario (CLAYSS)

\section{Resumen}

El aprendizaje servicio está teniendo una amplia difusión en el panorama internacional; si nos centramos en su desarrollo en el seno de la Educación Superior, más concretamente dentro del contexto Latinoamericano, indudablemente debemos hacer referencia a la universidad argentina. Una reciente estancia investigadora y formativa en la provincia de Buenos Aires nos ha permitido acercarnos a la realidad que diferentes universidades presentan en la inserción del aprendizaje servicio dentro de sus estructuras. La revisión de documentación institucional, la visita a diferentes proyectos y sobre todo, las entrevistas con diversos agentes implicados en su instauración y desarrollo, han sido de ayuda para entender el nivel de institucionalización que esta propuesta está alcanzando, así como los retos que se presentan a futuro. Todo esto se refleja en el análisis realizado y en unas conclusiones que recogemos a modo de cierre.

\section{Palabras clave}

Aprendizaje servicio, educación superior Argentina, extensión universitaria, responsabilidad social universitaria, institucionalización.

Fecha de recepción: 27/VII/2016

Fecha de aceptación: 27/II/2017 


\title{
Service learning in universities in Buenos Aires: moving towards institutionalization
}

\begin{abstract}
Service learning is having a wide spread in the international arena; if we focus on its development within Higher Education, more specifically in the Latin American context, we must undoubtedly refer to the Argentine University. A recent research and training stay in Buenos Aires, has allowed us to approach the reality that different universities are presenting on the inclusion of service learning within their structures. The review of institutional documentation, the visit of various projects and above all, the interviews with several agents involved in the establishment and development of it, have been helpful to understand the level of institutionalization that this proposal is achieving, as well as the challenges being presented to the future. All this is reflected in the analysis and the conclusions we recollect in the final closure.
\end{abstract}

\section{Keywords}

Service learning, Argentine Higher Education, University Extension, University Social Responsibility, Institutionalization. 


\section{Introducción}

En la región latinoamericana las prácticas de aprendizaje servicio son mucho más antiguas que el término mismo. El término aprendizajeservicio se ha difundido en el mundo desde la segunda mitad del siglo $X X$, según los matices culturales propios de cada región del planeta, donde se ha ido consolidando como una propuesta pedagógica con identidad propia. La larga tradición solidaria de los sistemas educativos y de las organizaciones sociales latinoamericanas, hace que muchas instituciones educativas (Escuelas y Universidades), sin tener conocimiento de los marcos teóricos y metodológicos ni denominarlas aprendizaje servicio, aún hoy desarrollen por iniciativa propia diferentes experiencias donde prima la articulación de los aprendizajes significativos con actividades solidarias al servicio de sus comunidades (Tapia, 2010). En todos los casos se observa que el objetivo final es lograr una educación que dé respuesta satisfactoria a demandas sociales inaplazables, con protagonismo juvenil que fortalece sus valores y vínculos con el otro (Pulfer, 2010).

Para los países latinoamericanos no se trata solamente de una propuesta pedagógica, sino una forma específica de intervención social que se suele denominar aprendizaje y servicio solidario. La fundamentación que da Tapia (2003) es que el concepto de solidaridad se interpreta como hacer juntos desde una acción colectiva por el bien común y para una ciudadanía activa, más que concebido como una acción altruista individual que es un concepto más afín a la cultura anglosajona. En esta región el contenido pedagógico y el contenido social de las prácticas de aprendizaje servicio son igualmente relevantes, reconociéndose el pensamiento de Paulo Freire en gran parte de estos fundamentos teóricos (Horton y Freire, 1990).

En cada país latinoamericano se pueden analizar las experiencias y programas desde su estructura, organización, formalidad y duración (Sherraden et al., 2006), desde su intensidad (BilligWeah, 2008) y desde propuestas que sistematizan la identificación de su grado de institucionalización (Furco, 2006). Así es que tanto las experiencias y programas como las formas de institucionalización comienzan a delinearse con un perfil propio, a partir de esta larga historia de prácticas educativas solidarias que resulta de interés identificar.

\section{La Universidad Argentina}

\subsection{Origen y evolución}

El surgimiento de las universidades en la América colonial española fue inspirado en el modelo de la Universidad de Salamanca, marcando su desarrollo hasta el avance del liberalismo, el positivismo y el idealismo y terminando de dar un vuelco con lo que se conoce como la Reforma Universitaria de 1918, reforma que se inicia en Córdoba (Argentina) y se expande por toda América Latina.

Sus inicios se remontan a la creación 
(1613) y su conversión en universidad de un colegio jesuita (1622) en la ciudad de Córdoba, momento en el cual otros 20 colegios fueron también convertidos en universidades para la formación de las elites que serían los futuros gobernantes. Con la expulsión de los jesuitas (1767) pasan a estar coordinadas por franciscanos. Después del inicio de los procesos independentistas de 1810 y con el objetivo acercar la formación de los jóvenes a Buenos Aires, lugar de radicación del nuevo gobierno, se crea en 1821 la Universidad de Buenos Aires, con la propuesta de ser un equilibrio entre los enfoques iluministas y la concepción tradicional. Esta propuesta fue de carácter provincial y estuvo sujeta a los momentos políticos del país hasta 1881 momento en la cual se la designa como universidad nacional (Fernández Lamarra, 2002). Recién en 1885 se reglamenta su funcionamiento mediante la sanción de la Ley 1597, conocida como Ley Avellaneda, que marca un inicio a los procesos de democratización que culminarán en la Reforma de 1918, con un nuevo sentido de responsabilidad social y política para la formación de los futuros profesionales.

La tradición solidaria se inicia en la Argentina en las universidades a partir de la Reforma Universitaria del año 1918 con el Movimiento Reformista de Córdoba (Argentina). Este movimiento emerge, en un reducto conservador, tradicionalista y católico que comenzó por un problema en apariencia menor, como fueron las exigencias de los estudiantes por una reforma en el sistema de provisión de las cátedras; y además para una mayor vinculación entre la universidad y la realidad del país. Sin embargo, muy pronto los reformistas advirtieron que el reclamo por un cambio en la universidad debía ser más bien un reclamo por una renovación de las estructuras sociales y políticas del país (Kandel, 2012). La Reforma del '18 se anticipó medio siglo al Mayo Francés y extendió su influencia a todas las universidades argentinas y latinoamericanas.

Se democratizaron los estatutos universitarios con base en los ejes del movimiento reformista, que fueron la autonomía universitaria, el co-gobierno estudiantil, los concursos docentes, la libertad y periodicidad de las cátedras, la investigación como función y la extensión universitaria como compromiso con la sociedad. Si bien la Reforma logró fortalecer la función social de la universidad, las actividades de vinculación adoptaron desde un inicio una postura asistencialista y paternalista.

\subsection{El papel de la Extensión universitaria}

En un inicio, la extensión universitaria fue entendida como una relación unidireccional de generación, divulgación y transferencia de conocimiento de la universidad hacia la sociedad. Recién en los años setenta, tanto en Argentina como en toda la región latinoamericana, esta postura empieza a ser cuestionada en favor de un nuevo concepto de extensión entendida como una relación bidireccional de compromiso, participación y transformación social 
(Lepore y Herrera, 2015).

Recién a fines de la década de 1980 e inicio de los años 90 se pueden comenzar a identificar la existencia de un enorme caudal de iniciativas solidarias espontáneas desarrolladas desde las escuelas y las instituciones de Educación Superior, con muy pocas políticas estatales al respecto, tanto en Argentina como en América Latina y el Caribe. Desde mediados de los noventa a la fecha, la promoción y fortalecimiento de una de estas iniciativas conocida como la pedagogía del aprendizaje servicio (A-S) se comienza a establecer como política pública y se observa que ha tenido un crecimiento exponencial que permitió su consolidación en la región (Ochoa, 2010). En Argentina, las propuestas educativas centradas en proyectos solidarios, estuvieron desde los años 80 y 90 concebidas principalmente para estudiantes y maestros de escuelas secundarias. Se destacan entre estas propuestas las reformas educativas de las provincias de Santa Fe (1983) y Buenos Aires (1997) y la creación del Programa Nacional Escuela y Comunidad (2000) que incorpora al A-S como un objetivo explícito de la política educativa. El programa Escuela y Comunidad, además de difundir y premiar las mejores prácticas, fue la base de la capacitación a nivel nacional para los docentes secundarios que tenían interés en aplicar estos proyectos en su práctica educativa. A partir del año 2003 la política pública de promoción del aprendizaje servicio del Ministerio de Educación de la Nación, se extiende a la educación superior, tanto desde el Programa Nacional Educación
Solidaria, como desde las líneas de financiamiento del llamado voluntariado educativo.

A nivel nacional es posible hoy identificar dos estrategias que han marcado el desarrollo de las estructuras de investigación colaborativa comunidad-universidad a través del enfoque de la educación solidaria: desde el plano pedagógico/educativo, las iniciativas de aprendizaje servicio impulsadas por el Ministerio de Educación; desde el lado de la investigación científica aplicada, los Programas de Desarrollo Tecnológico Social financiados por el Ministerio de Ciencia y Tecnología (Lepore y Herrera, 2015).

\section{La institucionalización del A-S en la Educación Superior}

\subsection{Práctica marginal Vs práctica institucionalizada}

Existen muchos trabajos que han sido desarrollados a través de la propuesta de A-S dentro del contexto de la Educación Superior. Proyectos unidos a materias concretas de una disciplina específica, proyectos unidos a diferentes disciplinas, otros relacionados con un Proyecto Fin de Carrera, Grado o Máster, etc. Sin embargo, a menudo, hemos de hablar de planteamientos que no tienen una continuidad (Martínez-Odriá, 2013; Sena, Baliño y Martínez, 2012). Esto puede ser debido a razones como la falta de financiamiento y/ o apoyo institucional; la discontinuidad en los contratos del profesorado universitario; la desaparición de entidades sociales 
con las que se colabora, etc.

Diferentes autores hacen referencia a la necesidad de que la intensidad y duración de los proyectos de A-S sean suficientes como para tener un impacto en la comunidad y en los propios estudiantes (Billig, 2000; Campo, 2014). La continuidad de un proyecto va a depender en gran medida del nivel de institucionalización de esa práctica en una entidad concreta. En relación a esta institucionalización y a la consideración de prácticas institucionalizadas de A-S, deberíamos atender a aquellos elementos que las caracterizan y que a su vez las diferencian de las prácticas marginales. Podemos observarlos en la siguiente tabla:

\begin{tabular}{|l|l|}
\hline \multicolumn{1}{|c|}{$\begin{array}{c}\text { La práctica } \\
\text { institucionalizada es: }\end{array}$} & $\begin{array}{c}\text { La práctica marginal } \\
\text { es: }\end{array}$ \\
\hline Rutinaria & Ocasional \\
\hline Extendida & Aislada \\
\hline Legitimada & No aceptada \\
\hline Esperada & Incierta \\
\hline Apoyada & Débil \\
\hline Permanente & Temporal \\
\hline Resiliente & Vulnerable \\
\hline
\end{tabular}

Tabla 1: Diferencias entre prácticas de A-S institucionalizadas y prácticas marginales. Fuente: adaptada de Furco $(2009,24)$.

3.2. La rúbrica para la evaluación de la institucionalización del A-S en la Educación Superior

Hablando de prácticas

institucionalizadas de A-S en el nivel universitario, es necesario mencionar a Andrew Furco y su rúbrica para la autoevaluación de la institucionalización de esta propuesta en la Educación Superior ${ }^{1}$.

Este trabajo se publicó inicialmente en 1998, y luego de su aplicación en ocho campus universitarios es revisado en 1999 por primera vez. El propio Furco ha realizado varias revisiones a posteriori, en los años 2002, 2003 y 2006. En la versión del 2006 se mantiene la misma estructura en las dimensiones propuestas, pero se actualiza parcialmente el lenguaje utilizado en las mismas (Furco, 2006).

La rúbrica está compuesta por 5 dimensiones, cada una de ellas incluye varios componentes, que son elementos sobre los cuales se evaluaría el grado de institucionalización de una universidad determinada.

En la siguiente tabla podemos observar la relación entre esas dimensiones y los componentes:

\begin{tabular}{|c|c|}
\hline DIMENSIONES & COMPONENTES \\
\hline $\begin{array}{l}\text { I. Filosofía y Misión } \\
\text { del aprendizaje } \\
\text { servicio }\end{array}$ & $\begin{array}{l}\text {-Definición de } \\
\text { aprendizaje servicio } \\
\text {-Plan estratégico } \\
\text {-Alineación con la misión } \\
\text { institucional } \\
\text {-Alineación con los } \\
\text { esfuerzos de reforma } \\
\text { educacional }\end{array}$ \\
\hline $\begin{array}{l}\text { II. Implicación y } \\
\text { apoyo de los } \\
\text { docentes en el } \\
\text { aprendizaje servicio }\end{array}$ & $\begin{array}{l}\text {-Sensibilización y } \\
\text { conocimiento por parte } \\
\text { de los docentes } \\
\text {-Implicación y apoyo de } \\
\text { docentes } \\
\text {-Liderazgo de docentes } \\
\text {-Incentivos y } \\
\text { reconocimientos a los } \\
\text { docentes }\end{array}$ \\
\hline
\end{tabular}

\footnotetext{
${ }^{1}$ Rúbrica completa en: http://www.tncampuscompact.org/files/sarubric. pdf
} 


\begin{tabular}{|c|c|}
\hline $\begin{array}{l}\text { III. Implicación y } \\
\text { apoyo de los } \\
\text { estudiantes en el } \\
\text { aprendizaje servicio }\end{array}$ & $\begin{array}{l}\text {-Sensibilización y } \\
\text { conocimiento de los } \\
\text { estudiantes } \\
\text {-Oportunidades para el } \\
\text { estudiantado } \\
\text {-Liderazgo del } \\
\text { estudiantado } \\
\text {-Incentivos y } \\
\text { reconocimientos a los } \\
\text { estudiantes }\end{array}$ \\
\hline $\begin{array}{l}\text { IV. Participación y } \\
\text { asociación de las } \\
\text { organizaciones } \\
\text { sociales o públicas } \\
\text { (comunidad) }\end{array}$ & $\begin{array}{l}\text {-Sensibilización y } \\
\text { conocimiento de la } \\
\text { contraparte comunitaria } \\
\text {-Entendimiento mutuo } \\
\text {-Liderazgo y voz de la } \\
\text { contraparte social o } \\
\text { pública }\end{array}$ \\
\hline $\begin{array}{l}\text { V. Apoyo } \\
\text { institucional al } \\
\text { aprendizaje servicio }\end{array}$ & $\begin{array}{l}\text {-Entidad coordinadora } \\
\text {-Entidad diseñadora de } \\
\text { políticas } \\
\text {-Equipo o personal } \\
\text {-Fondos y recursos } \\
\text {-Apoyo administrativo } \\
\text {-Apoyo de los } \\
\text { departamentos } \\
\text {-Evaluación y logro de } \\
\text { objetivos }\end{array}$ \\
\hline
\end{tabular}

Tabla 2: Dimensiones y componentes para evaluar el nivel de institucionalización del A-S en la Educación Superior. Fuente: adaptado de Furco $(2006,2)$.

Es importante destacar que esta rúbrica debiera ser adaptada a la idiosincrasia de aquella universidad en la que será aplicada. Esta aplicación a su vez permitirá determinar la fase de institucionalización que ésta alcanza en cada una de sus dimensiones. Cabe mencionar que en el estudio de los casos realizado, esta rúbrica ha orientado gran parte de los elementos estudiados.

3.3. Creciente interés por la promoción e institucionalización del A-S en la Universidad dentro del contexto latinoamericano
Furco $(2003,2006)$ y otros autores del ámbito anglosajón (Butin, 2006; Furco \& Holland, 2004; Harley, Harkavy y Benson, 2005; Prentice, 2002; Slater y Fotheringham, 2009) vienen desde hace tiempo trabajando en la incorporación del A-S a la Educación Superior, tanto en su promoción, como en la evaluación de sus impactos y en su institucionalización. El poder contar con estos referentes, la rápida expansión de la propuesta en nuestras universidades, y el afán por mejorar nuestras prácticas, entre otros, ha hecho que en el contexto latinoamericano cada vez surjan más puntos de encuentro donde hablar sobre A-S universitario e institucionalización. Veamos algunas muestras de ello.

\section{El surgir de redes, asociaciones y núcleos promotores}

En el contexto Iberoamericano la Red Ibero-Americana de aprendizaje servicio fue fundada en Buenos Aires el 29 de octubre de 2005, por un grupo de organismos gubernamentales, organizaciones de la sociedad civil, Universidades y organismos regionales de América Latina, Estados Unidos y España, que compartían el interés por promover al aprendizaje servicio como propuesta pedagógica, de protagonismo ciudadano y social de niños, adolescentes y jóvenes y como herramienta para la transformación de la realidad. La Red está liderada por CLAYSS (Centro Latinoamericano de Aprendizaje y Servicio Solidario, Argentina) y NYLC (National Youth Leadership Council, USA) y se constituye como un espacio de 
aprendizaje, de articulación, ejecución, sensibilización e investigación de oportunidades al desarrollo y apoyo para el aprendizaje servicio panamericano.

En el contexto español nos encontramos con la Red Española de Aprendizaje Servicio ${ }^{2}$, creada en el año 2010, y entre sus objetivos se encuentra el incidir en la formación, innovación, investigación, evaluación y difusión del A-S entre el profesorado universitario. Una de las líneas de trabajo primordiales está siendo su institucionalización en este ámbito.

En el mismo estado español nos encontramos con la creciente aparición de núcleos promotores del A-S dentro de la universidades, estos serían algunos ejemplos: El Grupo de $\operatorname{ApS}(U B)^{3}$ de la Universidad de Barcelona; GICE -Grupo de Investigación cambio educativo para la Justicia Social ${ }^{4}$ - de la Universidad Autónoma de Madrid, en el mismo hay una línea de trabajo específica sobre aprendizaje servicio y Justicia Social; el proyecto de Innovación Aprendizaje Servicio de la Universidad de Zaragoza5; etc.

En Chile nos encontramos con REASE Red de Aprendizaje y Servicio Chile ${ }^{6}$ - la

\footnotetext{
${ }^{2}$ https://sites.google.com/site/redapsuniversitari o/home

${ }^{3}$ http://www.ub.edu/grupapsub

${ }^{4}$ http://www.gice-uam.es/l\%C3\%ADneas-deinvestigaci\%C3\%B3n/aprendizaje-servicio-yjusticia-social

${ }^{5}$ http://www.unizar.es/aprendizaje_servicio/inde x.html

${ }^{6}$ https://reasechile.wordpress.com/
}

cual no es exclusivamente universitaria, pero donde la inmensa mayoría de miembros son pertenecientes a este ámbito, siendo uno de sus principales objetivos instalar el enfoque metodológico de aprendizaje servicio en distintas instituciones de educación, entre ellas, las universidades.

Foros de formación y reflexión sobre institucionalización del A-S en la Educación Superior

CLAYSS -Centro Latinoamericano de Aprendizaje y Servicio Solidario7- desde el año 2002 promueve la difusión del A$S$ ofreciendo programas de capacitación y de apoyo a universidades solidarias en diferentes países para llegar con acciones de capacitación a diferentes niveles. Además, produce materiales de apoyo, sostiene la investigación dentro de este ámbito, y organiza sistemáticamente el Seminario Internacional de A-S.

En febrero del 2013, la ACUP Asociación Catalana de Universidades Públicas- celebró un seminario ${ }^{8}$ en el que se presentó una ponencia sobre la institucionalización del aprendizaje servicio.

En el mismo 2013 en Barcelona, se celebraba la Conferencia GUNI -Global University Network for Innovationdonde también se presentó un taller ${ }^{9}$

\footnotetext{
${ }^{7}$ http://www.clayss.org.ar/02_apoyo/universidad es.htm

${ }^{8}$ http://www.acup.cat/es/noticia/la-acupcelebra-un-seminario-sobre-aprendizajeservicio\%E2\%80\%99-en-la-urv

${ }^{9}$ http://www.guninetwork.org/guni.conference/2 013-guni-conference/unis-
} 
sobre A-S e institucionalización.

Otro aporte lo encontramos en la Jornada de investigadores sobre A-S, que viene organizando CLAYSS en colaboración con diferentes organizaciones internacionales. Particularmente, el pasado 2015 se presentaron 2 trabajos $^{10}$ de investigación que hacían referencia explícita a la institucionalización del A-S en la Educación Superior, uno ligado a la Facultad de Educación de la Universidad de Barcelona, y otro a la Universidad del País Vasco (UPV/EHU).

Todo ello ha llamado nuestra atención y ha sido el motor para acercarnos a varias universidades en la Argentina, particularmente localizadas en la Ciudad de Buenos Aires y en el conurbano bonaerense, para realizar un pequeño estudio exploratorio y evaluar la situación en la que se encuentran.

\section{Hacia la institucionalización del A-S en algunas de las universidades argentinas}

\subsection{Casos de estudio}

Tal y como mencionábamos, en este estudio $^{11}$ nos hemos acercado a una serie de universidades, dos de ellas sitas en la Ciudad Autónoma de Buenos Aires y de alcance Nacional y otra en el

spain/?searchterm=institucionalizaci\%C3\%B3n \%20aprendizaje\%20-\%20servicio

10 http://www.clayss.org/3jornada/Libro_IIIJIA-

S.pdf

${ }^{11}$ En el contexto del conurbano Bonaerense otra universidad fue consultada, pero por razones ajenas a las autoras de este trabajo, no ha sido posible presentar la información recabada al respecto. conurbano bonaerense de la provincia de Buenos Aires.

\begin{tabular}{|c|c|c|}
\hline $\begin{array}{l}\text { UNIVERSIDAD } \\
\text { y/o FACULTAD }\end{array}$ & $\begin{array}{c}\text { TITULARIDAD } \\
y \\
\text { LOCALIZACIÓN }\end{array}$ & $\begin{array}{c}\text { MATRICULACIÓN } \\
\text { DE } \\
\text { ESTUDIANTES } \\
\end{array}$ \\
\hline $\begin{array}{l}\text { UNIVERSIDAD } \\
\text { DE BUENOS } \\
\text { AIRES -UBA- } \\
\text { (fundada el } 12 \\
\text { de agosto de } \\
1821 \text { ) }\end{array}$ & \multirow[t]{5}{*}{$\begin{array}{l}\text { Pública } \\
\text { Localizada en } \\
\text { la Ciudad } \\
\text { Autónoma de } \\
\text { Buenos Aires } \\
\text { con sedes } \\
\text { regionales } \\
\text { ubicadas en la } \\
\text { provincia de } \\
\text { Buenos Aires y } \\
\text { de alcance } \\
\text { nacional }\end{array}$} & $\begin{array}{l}\text { Según datos } \\
\text { recogidos en el } \\
\text { último censo } \\
\text { publicado } \\
(2004)^{12} \text {, la UBA } \\
\text { tiene una } \\
\text { matriculación } \\
\text { superior a los } \\
200.000 \\
\text { estudiantes }\end{array}$ \\
\hline $\begin{array}{l}\text { Facultad de } \\
\text { Odontología }\end{array}$ & & $\begin{array}{l}\text { Cerca de } 2.000 \\
\text { (según mismo } \\
\text { censo) }\end{array}$ \\
\hline $\begin{array}{l}\text { Facultad de } \\
\text { Ciencias } \\
\text { Veterinarias }\end{array}$ & & $\begin{array}{l}\text { Cerca de } 5.000 \\
\text { (según mismo } \\
\text { censo) }\end{array}$ \\
\hline $\begin{array}{l}\text { Facultad de } \\
\text { Arquitectura, } \\
\text { Diseño y } \\
\text { Urbanismo }\end{array}$ & & $\begin{array}{l}\text { Más de } 24.000 \\
\text { (según mismo } \\
\text { censo) }\end{array}$ \\
\hline $\begin{array}{l}\text { Facultad de } \\
\text { Ciencias } \\
\text { Económicas }\end{array}$ & & $\begin{array}{l}\text { Cerca de } 45.000 \\
\text { según mismo } \\
\text { censo) }\end{array}$ \\
\hline $\begin{array}{l}\text { UNIVERSIDAD } \\
\text { NACIONAL DE } \\
\text { LANÚS } \\
\text { (fundada el } 7 \\
\text { de junio de } \\
1995 \text { ) }\end{array}$ & $\begin{array}{l}\text { Pública } \\
\text { Localizada en } \\
\text { el Conurbano } \\
\text { Bonaerense }\end{array}$ & $\begin{array}{l}\text { Cerca de } 18.000 \\
\text { estudiantes }\end{array}$ \\
\hline $\begin{array}{c}\text { UNIVERSIDAD } \\
\text { TECNOLÓGICA } \\
\text { NACIONAL } \\
\text { (fundada el } 19 \\
\text { de agosto de } \\
1948 \text { ) }\end{array}$ & $\begin{array}{l}\text { Pública } \\
\text { Localizada en } \\
\text { la Ciudad } \\
\text { Autónoma de } \\
\text { Buenos Aires } \\
\text { con sedes } \\
\text { regionales } \\
\text { ubicadas en } \\
\text { todo el país }\end{array}$ & $\begin{array}{l}\text { Alrededor de } \\
70.000 \\
\text { estudiantes }\end{array}$ \\
\hline
\end{tabular}

Tabla 3: Universidades estudiadas. Algunos datos. Fuente: elaboración propia.

A continuación se detallan tanto la ${ }^{12}$ http://www.uba.ar/institucional/censos/Estudia
$\underline{\text { ntes2004/censo-estudiantes.pdf }}$ 
presencia del aprendizaje servicio como el grado de institucionalización en las mismas.

\section{UNIVERSIDAD DE BUENOS AIRES - UBA-}

Como hemos podido observar, la Universidad de Buenos Aires es una institución de gran tradición, tiene casi 200 años de antigüedad, ésta representa un espacio formativo de reconocido prestigio y de un marcado compromiso con la sociedad argentina.

El Ministerio de Educación de la Nación Argentina convocó en los años 2004, 2006, 2008 y 2010, un Premio Presidencial en el contexto de las Prácticas Educativas Solidarias en la Educación Superior ${ }^{13}$, cabe señalar que la Universidad de Buenos Aires recibió premios destacados en todas sus ediciones (Kandel, 2012).

Esta universidad ha ido dando pasos en la incorporación del A-S en su seno, hasta el punto de que su Consejo Superior ${ }^{14}$ haya resuelto establecer la obligatoriedad de las prácticas sociales educativas como requisito para que el alumnado pueda egresarse en la misma a partir del año académico 2017. En el análisis que presentaremos en adelante, veremos el nivel de adaptación que las Facultades estudiadas han podido alcanzar.

Facultad de Odontología

\footnotetext{
${ }^{13}$ http://www.me.gov.ar/edusol/premios_preside nciales.html

${ }^{14}$ http://www.uba.ar/archivos_uba/2014-0423_172.pdf
}

En el año 1979 la profesora Noemí Bordoni pone en marcha la Cátedra de Odontología preventiva y comunitaria, a partir del año 1985, en la misma se integran prácticas sociales curriculares que se encuadran dentro de la propuesta del A-S (Bordoni y Squassi, comunicación personal, 27 de julio, 2015; Squassi, 2011). Esta cátedra ha tenido diferentes nombres a lo largo de estos años, pero siempre ha mantenido su esencia. Se comenzó trabajando en lo individual (apoyo odontológico uno a uno), para después pasar a una intervención más en lo colectivo (en escuelas y organizaciones). A través de estos proyectos se propone un trabajo en intervención, investigación y divulgación.

En la actualidad estas prácticas se articulan mediante las asignaturas Articulación docencia y servicio de salud, de $5^{\circ}$ curso, y Epidemiología, de 40 (Bordoni y Squassi, comunicación personal, 27 de julio, 2015).

\section{Facultad de Ciencias veterinarias}

La Facultad de Ciencias Veterinarias también aporta una amplia experiencia en el desarrollo de acciones encuadradas en la propuesta del A-S, viene desarrollando este tipo de prácticas desde finales de los años 90 (Coppola, 2012; Miguez, Checchia, Vaccaro, Barboni y Grinsztajn, 2012).

Algunos de los ejemplos que podemos encontrar son Agua para todos y para todo, planificado y desarrollado por la cátedra de Bases Agrícolas (19962003), su origen se sitúa en el interés por vincular la enseñanza universitaria, 
con los resultados en investigación científica y la transferencia a la comunidad. A lo largo de estos años la cátedra ha centrado sus esfuerzos en la docencia e investigación referida al manejo sostenible de recursos agropecuarios, dentro de esta línea de trabajo, se viene interviniendo en aspectos relacionados con la gestión del agua en entornos rurales (Herrera, 2011).

En el proyecto Los Piletones los estudiantes de Veterinaria participan en un proyecto que se desarrolla en el contexto de Villa Soldati desde el año 2006, se trata de un contexto con una población carenciada y multicultural. Las actividades desarrolladas por el alumnado resultan ser prácticas preprofesionales relacionadas, entre otras, con el diagnóstico, la prevención y el control de las enfermedades que se transmiten de los animales al hombre; la tenencia responsable de animales y el control poblacional de mascotas. Las tareas realizadas por este alumnado se desempeñan en un contexto que propicia el desarrollo de valores, actitudes prosociales y competencias genéricas y específicas de la titulación de veterinaria (Martínez Vivot y Folgueiras, 2012). Este proyecto viene implementándose en estrecha colaboración y coordinación con la Fundación Margarita Barrientos ${ }^{15}$, que opera en la Villa.

El proyecto El camino de la lana, desarrollado desde el año 2000 por la cátedra de Producción de Ovinos, en respuesta a la crisis del sector y con el

\footnotetext{
${ }^{15}$ http://www.margaritabarrientos.com.ar/
}

fin de hacer un aporte directo al pequeño productor (de 5 a 50 ovejas), viene desarrollando diversas actividades de extensión. Éstas se relacionan, entre otras, con la identificación de necesidades relacionadas con la producción ovina en el contexto rural; la capacitación de productores; o el asesoramiento en el procesamiento artesanal de la lana. En el año 2006, surgió la propuesta de presentar un proyecto orientado a pequeñas comunidades del área rural de San Andrés de Giles. Con el tiempo también se ha llegado a otras comunidades como San Andrés y Baradero, entre otras (Coppola, 2012)

La integración del A-S en este caso se hace mediante la asignatura de Sociología en los primeros tramos de la carrera, y mediante su correlativa, el Taller de Sociología Rural y Urbana y Prácticas Solidarias, todo ello permite que el alumnado tenga una intervención directa en el ámbito socio comunitario, $y$ en contextos productivos.

Facultad Arquitectura, Diseño y Urbanismo

En la Facultad de Arquitectura, Diseño y Urbanismo (FADU) encontramos dos interesantísimas propuestas ligadas al aprendizaje servicio El Seminario Interdisciplinario para la Urgencia Social -SIUS-, y el Taller Libre de Producción Social -TLPS-

El SIUS (Marconi y Frid, 2011) es una materia electiva en el currículo oficial para alumnos avanzados de las 6 carreras que integran esta Facultad (Arquitectura, Diseño Gráfico, Diseño 
industrial, Diseño de indumentaria y textil, Diseño de imagen y sonido, Diseño de planificación de paisaje). También está abierto mediante convenio a estudiantes de otras universidades latinoamericanas y europeas.

El proyecto comenzó en el 2002 con el Ciclo Básico Común ${ }^{16}$ (que todo aspirante a graduarse en la UBA debe superar); y a día de hoy sigue desarrollándose a través de proyectos ${ }^{17}$ en colaboración con colectivos, entidades de diversos ámbitos como villas, centros de salud mental, granjas comunitarias, escuelas, bibliotecas, etc.

TLPS se trata de una cátedra libre que "desarrolla actividades de FORMACIÓN para el EJERCICIO PROFESIONAL en el hábitat, comprometido con los intereses y necesidades populares" (Red ULACAV, $\mathrm{s} / \mathrm{f})$. EI TLPS fue aprobado como Cátedra Libre Interdisciplinaria de la FADU en 2006. Esta cátedra articula formación, investigación y extensión, a través de proyectos ${ }^{18}$ en los que se involucran estudiantes, docentes y organizaciones sociales. La integración curricular se orienta a través de un enfoque proyectual interdisciplinario que integra, entre otras, disciplinas del diseño, la psicología social, o la antropología social y económica. Los estudiantes de la FADU en condiciones de realizar pasantías de extensión ( 2 materias de la carrera aprobadas) pueden obtener créditos equivalentes a

\footnotetext{
16 http://www.cbc.uba.ar/HomePage

${ }^{17}$ http://www.sius.com.ar/proyecto-2015.html\#

18 http://www.tlps.com.ar/elaboracionespropias/cuadernillos-de-experiencias/
}

una materia electiva de 60 horas por cada cuatrimestre cursado.

\section{Facultad de Ciencias Económicas}

Desde el 2006 en esta Facultad se vienen desarrollando diferentes actividades en las que el vínculo con el medio es muy fuerte, la Secretaría de Extensión Universitaria de la misma, y en el marco de la Responsabilidad Social Universitaria (RSU), siempre ha tenido claro que era esencial consolidar este vínculo (PVU, 2012).

A partir del segundo cuatrimestre del 2010, se pone en marcha la materia Cátedra de Honor Dr. Bernardo Kliksberg Prácticas para la Inclusión Social. Se trata de una asignatura optativa que puede ser elegida por alumnado perteneciente a cualquiera de las carreras que se dictan en la Facultad (Licenciatura en Economía, Licenciatura en Administración, Contador Público, Licenciatura en Sistemas y Actuario). El hecho de integrar a estudiantes de estas cinco carreras permite que desde esta materia se de un abordaje interdisciplinario a las problemáticas que se pretende atajar desde los diferentes proyectos. Desde el año 2010, cerca de 800 estudiantes habrán pasado por este tipo de proyectos (Bertevello, comunicación personal, 24 de agosto, 2015). Algunas de las iniciativas impulsadas son Apoyo y Fortalecimiento a organizaciones de la Economía Social o Economía en los barrios (PVU, 2012).

UNIVERSIDAD NACIONAL DE LANÚS

En el año 2007 se pusieron en marcha 
unos programas de verano donde jóvenes de entornos desfavorecidos se acercaban a la universidad. Se comenzó a hacer un trabajo voluntario en pro de los derechos de los chicos, también se inició un trabajo con los profesionales que trabajan con ellos. Esta iniciativa ha ido madurando hacia programas permanentes como la UNLA de los jóvenes ${ }^{19}$.

Todos los proyectos de aprendizaje servicio y actividades de voluntariado estudiantil de esta universidad dependen de la Dirección de Cooperación ${ }^{20}$. La misma desarrolla lo que son llamados Programas sociocomunitarios (Micele, comunicación personal, 11 de agosto, 2015).

El alumnado de la UNLA se puede enrolar en estos programas de forma voluntaria, mediante prácticas pre profesionales, o en relación a asignaturas concretas. En lo que al A-S se refiere, es la Secretaria académica quien se encarga de revisar la participación de docentes y alumnado, de valorar y validar si efectivamente existe un proyecto educativo, etc. La secretaria de administración revisa la adecuación de recursos, equilibrio, en relación al encargo.

\section{UNIVERSIDAD TECNOLÓGICA NACIONAL}

19

http://www.unla.edu.ar/index.php/noticias/199novedades-destacadas/3308-comienza-elprograma-jovenes-lideres-comunitarios

${ }^{20}$ http://www.unla.edu.ar/index.php/secretariade-cooperacion-y-servicio-publico
La Universidad Tecnológica Nacional UTN-, creó en 2010 el llamado Programa de Responsabilidad Social Universitaria (PRORSU) ${ }^{21}$, el objetivo principal está orientado a proporcionar un espacio de reflexión sobre el rol de la Universidad actual. En el 2012 el PRORSU incorpora algunas herramientas que permiten desarrollar un modelo de voluntariado universitario que integra las acciones sociales más tradicionales, con objetivos pedagógicos y de construcción de conocimiento.

Cada una de las facultades tiene su espacio para desarrollar este tipo de proyectos. Las iniciativas que se ofertan desde las cátedras, se pueden integrar en materias optativas, en materias obligatorias, también en prácticas preprofesionales (Berenblum, comunicación personal, 6 de agosto, 2015). En este contexto, el A-S se entiende como una estrategia para el desarrollo de la Responsabilidad Social Universitaria.

Un ejemplo de los diversos proyectos liderados en la UTN lo tenemos en Bahía Blanca (provincia de Buenos Aires), se trata de una propuesta que tiene como propósito estrechar la brecha digital en la escuela rural N041 Alférez San Martín. La localización de esta escuela y la orografía que le rodea hacía que hubiera quedado fuera del acceso a la red. Docentes y alumnos de la Cátedra Comunicaciones II, de la carrera de ingeniería eléctrica hicieron posible el acceso a Internet para esa

\footnotetext{
${ }^{21}$ http://www.utn.edu.ar/secretarias/administrati va/prorsuantecedentes.utn
} 
escuela ${ }^{22}$.

\subsection{Procedimiento}

El estudio de los casos presentados previamente se ha desarrollado a través de:

- La consulta de diversa documentación como papers, comunicaciones de experiencias y documentación institucional

- entrevistas con agentes que tienen una amplia experiencia y conocimiento del estado del aprendizaje servicio en el contexto de la Educación Superior, y más concretamente, dentro del contexto de las universidades argentinas

- entrevistas a docentes y personal de administración referentes en diferentes universidades, en relación al desarrollo del $A-S$ en éstas

- la visita a algunos proyectos de A-S que se están desarrollando en las mismas.

El acceso a la inmensa mayoría de informantes y proyectos ha sido facilitado por CLAYSS. Las entrevistas se desarrollaron entre el 6 de julio y el 31 de agosto del 2015. En la siguiente tabla podemos observar cuáles fueron los principales elementos de estudio.

1. Nivel de institucionalización del A-S

2. Inicios del A-S en la Escuela/Facultad o Universidad

3. Hitos en el desarrollo del A-S en la Escuela/Facultad o Universidad

4. Articulación del A-S en la Escuela/Facultad o Universidad

${ }^{22}$ http://www.frbb.utn.edu.ar/utec/utec/30/n1.ht $\mathrm{ml}$
5. Concepción del A-S en Escuela/Facultad o Universidad

6. Objetivos que se marcan mediante el desarrollo del A-S

7. Papel del alumnado y SSCC en la promoción y desarrollo del A-S

8. Retos en la promoción, desarrollo y sostenibilidad del A-S en la Escuela/Facultad o Universidad

Tabla 4: Elementos de estudio. Fuente: elaboración propia

\subsection{Algunos resultados y discusión}

Tal y como hemos podido ver, las entrevistas realizadas en las diferentes universidades a las que nos dirigimos, permitieron indagar en varios elementos relacionados con el desarrollo del A-S en las mismas, sin embargo, presentaremos a continuación algunos de los resultados encontrados. Nos referiremos a los siguientes elementos:

El nivel de institucionalización/consideración del A$S$ dentro de la Universidad/Facultad, entendido como nivel de instauración, formalización de la misma (inclusión en planes estratégicos, etc.) y reconocimiento institucional.

La dependencia de las actividades de A$S$ haciendo referencia a la estancia universitaria, paraguas bajo el que se promueven y desarrollan.

Apoyos institucionales como estructura que permite un planteamiento, desarrollo y evaluación adecuada de las iniciativas que se presentan. Hablamos de la dotación de recursos personales, económicos, etc.

Papel del alumnado y de los Socios Comunitarios. En términos de protagonismo de los estudiantes, y de 
esas entidades sociales, colectivos, etc. con quienes colaboramos a través de nuestros proyectos, su nivel de participación en todo lo relacionado con la instauración de los proyectos de A-S.

Retos que se prevén a futuro como principales obstáculos a salvar en la promoción, desarrollo y sustento del AS.

La documentación revisada, las entrevistas realizadas, y el análisis de las mismas, nos ha permitido ver que, a pesar del innegable desarrollo que el AS está teniendo en estas universidades, está lejos de alcanzar un nivel de institucionalización, cuestión que podemos derivar si ponemos en diálogo los elementos estudiados principalmente y la rúbrica que nos propone Furco (2006). Hablaríamos de la necesidad de un mayor desarrollo en la inmensa mayoría de las dimensiones que presenta. Esto nos hace recordar que la verdadera institucionalización del A-S requiere de una reestructuración radical, una reordenación todos los recursos que componen nuestra institución (Harley, Harkavy y Benson, 2005), la necesidad está ahí, pero no siempre existen facilidades para ello.

\section{Conclusiones}

La revisión de documentación diversa, la visita a diferentes proyectos de A-S y las entrevistas mantenidas con diferentes colegas inmersos en el desarrollo de este tipo de iniciativas, nos ha permitido verificar que el $A-S$ es un importante aliado a la hora de promover procesos que integren los ámbitos de docencia, extensión e investigación en la universidad, ámbitos que a menudo se presentan fragmentados (Ierullo, en prensa). Sin embargo, también hemos podido observar que, a pesar de los años de experiencia en el desarrollo del A-S dentro de la universidad de la Argentina, al menos, en los casos estudiados dentro de la ciudad y de la provincia de Buenos aires, los logros alcanzados en términos de institucionalización son bien distintos y en alguno de los casos, podríamos denominar pobres.

Sólo una de las universidades estudiadas recoge la obligatoriedad del cumplimiento de las prácticas sociales para que su alumnado pueda egresarse, este es el caso de la UBA, la cual además resulta ser la institución más antigua a la que nos hemos acercado. Debemos añadir que todavía no ha podido darse un desarrollo efectivo de la resolución al respecto, es más, según lo que diferentes agentes nos han transmitido, su estructura parece no estar del todo preparada para una implantación masiva de la propuesta (formación del profesorado, financiación, ordenación curricular...), lo cual presenta un interesante elemento para el debate sobre los modelos de implantación del A-S en la Educación Superior.

En relación a la dependencia de la actividad de $A-S$ respecto a las diferentes instancias que componen la institución, veíamos como entre los casos estudiados se daba diversidad de dependencias, en unos casos el A-S colgaba de instancias más unidas al ámbito de Extensión, en otros del de 
Docencia, incluso de una articulación entre ambos. Entendemos que la dependencia de una u otra instancia puede acarrear implicaciones bien distintas respecto al papel que esa universidad otorga a esta propuesta; al apoyo que pueden tener los diferentes agentes implicados; el nivel de financiamiento que se aporte, entre otras. Hay quien podría pesar que al tratarse de una propuesta pedagógica ésta necesariamente debiera depender de instancias relacionadas con la docencia. También somos conscientes de que el desarrollo del A-S dentro de una maquinaria tan compleja como es la universidad, exige, cuanto menos, justifica, cierta flexibilidad en los modelos de implementación.

En relación al papel protagonista del estudiantado y de los socios comunitarios en el marco de implementación del A-S universitario, hemos podido ver como en todos los casos se daba una presencia y un protagonismo notorio, destacando especialmente la articulación con y el apoyo al alumnado, aspecto que consideramos de absoluto relieve en el seno de una práctica de base experiencial y orientada al desarrollo de la responsabilidad social universitaria.

Todos los casos estudiados han presentado diversidad de apoyos en la implementación del A-S, apoyos como la capacitación docente; la financiación a través de varias vías; o la sensibilización a diferentes agentes implicados en los proyectos de A-S. Sin embargo, a tenor de las voces recogidas, existen importantes retos de cara a la institucionalización y
RIDIS:

sostenibilidad del A-S en las instituciones.

Todas las personas entrevistadas apuntaban importantes retos de futuro entre los que destacan la evaluación del impacto que está teniendo el A-S en la comunidad; la necesidad de coordinación entre diferentes instancias involucradas en la implementación del A-S dentro de la universidad; el estudio de la viabilidad de una aplicación masiva, a nivel macro dentro de la institución; y las posibilidades de continuidad de los proyectos, posibilidades unidas a cuestiones económicas o de otra índole.

Entendemos que este estudio ha permitido evidenciar algunos claros y oscuros de lo que está siendo el desarrollo del A-S en la Argentina, sin embargo, somos conscientes de las limitaciones del mismo, limitaciones unidas principalmente al reducido número de casos al que hemos podido acceder, y a la idiosincrasia de los mismos ya que en este caso presentado son todas universidades estatales. Por ejemplo fue nuestra intención acceder a una universidad privada, pero no nos fue posible ya que luego de obtener la información no pudimos presentar los resultados por decisión de la propia universidad. Todo ello nos hace apuntar la necesidad de seguir avanzando en estudios que recojan otros testimonios y que evidencien los avances que han podido darse en los casos aquí presentados.

\section{Referencias bibliográficas}

Billig, S. (2000). Research on K-12 
school-based service-learning: The evidence builds. Phi Delta Kappan, 81(9), 658-664. Recuperado de http://digitalcommons.unomaha.edu/cg i/viewcontent.cgi?article $=1003 \&$ context $=$ slcek 12

Billig-Weah (2008). K-12 Servicelearning Standards for Quality Practice. En National Youth Leadership Council (2008). Growing to Greatness. St. Paul, MN: University of Minnesota.

Butin, D.W. (2006). The limits of service-learning in Higher Education. The review of Higher Education, 29 (4), 473-498. Recuperado de http://tag.ubc.ca/wpcontent/uploads/2008/01/thelimitsofser vicelearninginhighereducation.pdf

Campo, L. (2014). Aprendizaje servicio y educación superior Una rúbrica para evaluar la calidad de proyectos (Tesis doctoral inédita). Departamento de Teoría e Historia. Universidad de Barcelona.

Coppola, M. (2012). El camino de la lana: prácticas de Aprendizaje-Servicio aplicado al desarrollo rural. II Jornada de investigadores en aprendizajeservicio. Buenos Aires: CLAYSS-Red Iberoamericana de AprendizajeServicio. Recuperado de http://www.clayss.org.ar/06_investigaci on/jornadas/Libro_IIJIAS_COMPLETO.pdf

Fernández, N. (2012). La educación superior en América Latina. Aportes para la construcción de una nueva agenda. Debate Universitario, 1 (1), 129. Recuperado de: http://ppct.caicyt.gov.ar/index.php/deb ate-universitario/article/view/16039357-5.pdf

Furco, A. (2003). Issues of definition and program diversity in the study of Service-Learning. En S.H. Billig \& A.S. Waterman (Eds.) Studying ServiceLearning. Innovations in Education Research Methodology. New York: Routledge.

Furco, A. (2006). Self-Assessment Rubric for the Institutionalization of Service-Learning in Higher Education. Berkeley: University of California. Service-Learning Research \& Development Centre. Recuperado de http://www.tncampuscompact.org/files/ sarubric.pdf

Furco, A. (2009). Institutionalizing Service-Learning in Higher Education. University of Minnesota: Office for Public Engagement. Recuperado de http://tncampuscompact.org/files/furco .pdf

Furco, A. y Holland, B. (2004). Institutionalizing Service learning in higher education: Issues and strategies for chief academic officers. En M. Langseth y S. Dillon. (Eds.). Public work and the academy. Bolton, M.A.: Anker Publishing Company.

Harley, M., Harkavy, I. y Benson, L. (2005). Putting down roots in the groves of academy: The challenges of institutionalizing service-learning. En D.W. Butin. Service-learning in Higher Education. New York: Palgrave Macmillan. 
Horton, M. y Freire, P. (1990). We Make the Road by Walking: Conversations on Education and Social Change.

Philadelphia: Temple University Press.

Ierullo, M. (en prensa). El aprendizaje y servicio solidario en las universidades. Informe de evaluación del Programa de Apoyo y Fortalecimiento para Proyectos de Aprendizaje-Servicio Solidario en Universidades 2012-2015. Buenos Aires: CLAYSS.

Kandel, V. (2012). Estudiantes y ciudadanos: La universidad de Buenos Aires como espacio de formación de ciudadanía (Tesis doctoral inédita). Facultad de Filosofía y Letras. Universidad de Buenos Aires.

Martínez-Odriá, A. (2103). Experiencias de Aprendizaje Servicio en la Universidad San Jorge: Primeros pasos. En L. Rubio, E. Prats y L. Gómez (Coord.) Universidad y Sociedad. Experiencias de Aprendizaje Servicio en la Sociedad. Recuperado de http://hdl.handle.net/2445/46344

Martínez Vivot, M. y Folgueiras, P. (2012). Competencias genéricas y específicas adquiridas por estudiantes de veterinaria en un proyecto de Aprendizaje-Servicio. II Jornada de investigadores en aprendizaje-servicio.

Buenos Aires: CLAYSS-Red Iberoamericana de AprendizajeServicio. Recuperado de http://www.clayss.org.ar/06_investigaci on/jornadas/Libro_IIJIAS_COMPLETO.pdf

Miguez, M., Checchia, B., Vaccaro, M., Barboni, A.M. y Grinsztajn, F. (2012).
Proyectos solidarios. Currículum, compromiso social, y gestión del conocimiento. Buenos Aires: FCV-UBA EUDEBA.

Ochoa, E.M. (2010). AprendizajeServicio en América Latina: apuntes sobre pasado y presente. Tzhoecoen, 3 (5), 108-125.

Prentice, M. (2002). Institutionalizing Service Learning in Community Colleges. American Association of Community Colleges. Recuperado de http://www.aacc.nche.edu/Resources/a accprograms/horizons/Documents/pren tice_rb.pdf

Pulfer, D. (2010) Reflexiones y presentación. Tzhoecoen, 3 (5), 11-12.

Programa de Voluntariado Universitario. (2012). Manual de Voluntariado.

Buenos Aires: Facultad de Ciencias Económicas. Universidad de Buenos Aires. Recuperado de http://voluntariadofce.econ.uba.ar/wpcontent/uploads/2011/06/MANUALVOLUNTARIADO.pdf

Red Universitaria Latinoamericana de cátedras de Vivienda. (s/f). Boletín 2. Recuperado de http://blog.ucc.edu.ar/ssh/files/2015/0 4/BOLETIN-02-Red-ULACAV.pdf

Sena, S., Baliño, G. y Martínez, M. (2012). En un diálogo de saberes: organizaciones sociales, universidad, economía social y solidaria. En M.A. Herrero y M.N, Tapia (Comp.) Actas de la II Jornada de investigadores en aprendizaje-servicio. Buenos Aires: CLAYSS-Red Iberoamericana de 
aprendizaje-servicio. Recuperado de http://www.clayss.org.ar/06_investigaci on/jornadas/Libro_IIJIAS_COMPLETO.p $\mathrm{df}$

Sherraden, M. S.; Stringham, J.; Sow, S. C. y McBride, A. M. (2006). The forms and structure of international voluntary service. Voluntas, $17,163-$ 180.

Squassi, A. (2011). Cátedra de Odontología Preventiva Y Comunitaria. XI Congreso Iberoamericano de Extensión universitaria. Las Prácticas Sociales Educativas como abordaje para la resolución de los problemas comunitarios: casos para el análisis. Buenos Aires: Universidad de Buenos Aires. Recuperado de http://www.unl.edu.ar/iberoextension/d vd/archivos/ponencias/mesa3/laspracticas-sociales-educa.pdf

Stater, K.J. y Fotheringham, E. (2009). Mechanisms for institutionalizing service-learning and community partner outcomes. Journal of Higher Education Outreach and Engagement, 13 (2), 730.

Tapia, M. N. (2003). Servicio y

Solidaridad en Español: Una cuestión terminológica o un problema conceptual. En H. Perold, M. Sherraden, and S. Stroud (Eds), Servicio Cívico y Voluntariado. El Servicio Cívico y el Voluntariado en el Siglo XXI Johannesburg: Global Service Institute, USA-Volunteer and Service Enquiry Southern Africa.

Tapia, M. N. (2010). La propuesta pedagógica del aprendizaje-servicio: una perspectiva latinoamericana.

Tzhoecoen, 3 (5), 23-44.

\section{AGRADECIMIENTOS}

Queremos hacer llegar nuestro más sincero agradecimiento a todas aquellas personas que de forma desinteresada han compartido su tiempo y experiencias, $y$ de un modo $u$ otro han colaborado en el desarrollo de este trabajo: Marcelo Miguez, Noemi Bordoni, Aldo Squassi, Juliana Bertevello, Carla Micele, Daniel Berenblum, Juan Frid, Martín Ierullo, María Nieves Tapia y Enrique Ochoa 